\title{
Molecular phylogeny of mulberries reconstructed from ITS and two cpDNA sequences
}

\author{
Yahui Xuan $^{1}$, Yue Wu ${ }^{1}$, Peng Li ${ }^{1}$, Ruiling Liu ${ }^{1}$, Yiwei Luo ${ }^{1}$, Jianglian Yuan ${ }^{1}$, Zhonghuai Xiang ${ }^{1}$, Ningjia He $^{\text {Corresp. }}{ }^{1}$ \\ ${ }^{1}$ State Key Laboratory of Silkworm Genome Biology, Southwest University, Chongqing, China \\ Corresponding Author: Ningjia He \\ Email address: hejia@swu.edu.cn
}

Background: Species in the genus Morus (Moraceae) are deciduous woody plants of great economic importance. The classification and phylogenetic relationships of Morus, especially the abundant mulberry resources in China, is still undetermined. Internal transcribed spacer (ITS) regions are among the most widely used molecular markers in phylogenetic analyses of angiosperms. However, according to the previous phylogenetic analyses of ITS sequences, most of the mulberry accessions collected in China were grouped into the largest clade lacking for phylogenetic resolution. Compared with functional ITS sequences, ITS pseudogenes show higher sequence diversity, so they can provide useful phylogenetic information.

Methods: We sequenced the ITS regions and the chloroplast DNA regions TrnL-TrnF and TrnT-TrnL from 33 mulberry accessions, and performed phylogenetic analyses to explore the evolution of mulberry.

Results: We found ITS pseudogenes in 11 mulberry accessions. In the phylogenetic tree constructed from ITS sequences, clade B was separated into short-type sequence clades (clades 1 and 2), and a longtype sequence clade (clade 3 ). Pseudogene sequences were separately clustered into two pseudogroups, designated as pseudogroup 1 and pseudogroup 2 . The phylogenetic tree generated from cpDNA sequences also separated clade $B$ into two clades.

Conclusions: Two species were separated in clade B. The existence of three connection patterns and incongruent distribution patterns between the phylogenetic trees generated from cpDNA and ITS sequences suggested that the ITS pseudogene sequences connect with genetic information from the female progenitor. Hybridization has played important roles in the evolution of mulberry, resulting in low resolution of the phylogenetic analysis based on ITS sequences. An evolutionary pattern illustrating the evolution history of mulberry is proposed. These findings have significance for the conservation of local mulberry resources. Polyploidy, hybridization, and concerted evolution have all played the roles in the evolution of ITS sequences in mulberry. This study will expand our understanding of mulberry evolution. 
2 Molecular phylogeny of mulberries reconstructed from ITS and two cpDNA

3 sequences

4

5

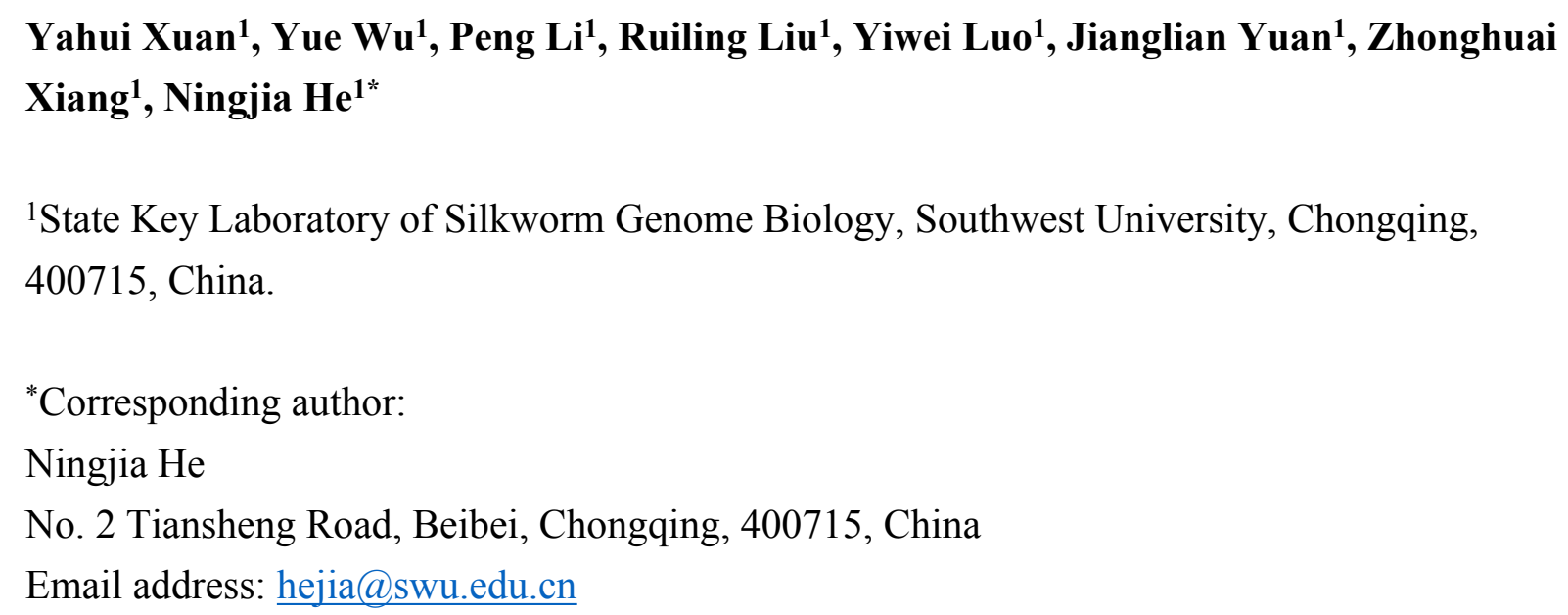

\section{Abstract}

Background: Species in the genus Morus (Moraceae) are deciduous woody plants of great economic importance. The classification and phylogenetic relationships of Morus, especially the abundant mulberry resources in China, is still undetermined. Internal transcribed spacer (ITS) regions are among the most widely used molecular markers in phylogenetic analyses of angiosperms. However, according to the previous phylogenetic analyses of ITS sequences, most of the mulberry accessions collected in China were grouped into the largest clade lacking for phylogenetic resolution. Compared with functional ITS sequences, ITS pseudogenes show higher sequence diversity, so they can provide useful phylogenetic information.

Methods: We sequenced the ITS regions and the chloroplast DNA regions $\operatorname{TrnL-TrnF}$ and $\operatorname{Trn} T$ - 
$27 \operatorname{Trn} L$ from 33 mulberry accessions, and performed phylogenetic analyses to explore the

28 evolution of mulberry.

29 Results: We found ITS pseudogenes in 11 mulberry accessions. In the phylogenetic tree constructed from ITS sequences, clade B was separated into short-type sequence clades (clades 1 and 2), and a long-type sequence clade (clade 3). Pseudogene sequences were separately clustered into two pseudogroups, designated as pseudogroup 1 and pseudogroup 2. The phylogenetic tree generated from cpDNA sequences also separated clade B into two clades.

Conclusions: Two species were separated in clade B. The existence of three connection patterns and incongruent distribution patterns between the phylogenetic trees generated from cpDNA and ITS sequences suggested that the ITS pseudogene sequences connect with genetic information from the female progenitor. Hybridization has played important roles in the evolution of mulberry, resulting in low resolution of the phylogenetic analysis based on ITS sequences. An evolutionary pattern illustrating the evolution history of mulberry is proposed. These findings and concerted evolution have all played the roles in the evolution of ITS sequences in mulberry. This study will expand our understanding of mulberry evolution.

Keywords: Morus; internal transcribed spacer; pseudogenes; concerted evolution; phylogenetic analyses

\section{Introduction}


48 Mulberries (Morus spp.), in the family Moraceae (order Rosales), are deciduous woody plants of

49 great economic importance. The leaves of mulberry are the main food for silkworms (Sánchez

50 2002). The fruit of Morus species also has nutritional and medicinal value (Chen et al. 2016b;

51 Priya 2012). Mulberry is believed to have originated in the Himalayan foothills and spread

52 across the Eurasian, American, and African continents (Nepal \& Ferguson 2012; Vijayan et al.

53 2004). In 1753, Linnaeus assigned seven species in the genus Morus based on the color of

54 aggregate fruits, leaf shape, and the presence of a cuticle (Linnaeus, 1753). Since then, many

taxonomists have revised the classification of Morus (Bureau, 1873; Koidzumi, 1917; Zhou and

Gilbert, 2003), and 10-16 species are currently recognized (Nepal \& Ferguson 2012; Sanchez

2002). Spontaneous and artificial hybridization can occur between different species of mulberry,

leading to successive interspecific characteristics (Botton et al. 2005). The continuous variations

in the phenotypic characteristics have made it difficult to classify mulberry resources (Vijayan et

al. 2004). Consequently, the classification of mulberry remains highly controversial.

Compared with phenotypic characters, DNA-based molecular markers represent a faster and more reliable system for germplasm characterization and phylogenetic analyses. Another advantage of DNA-based molecular markers is that they are not influenced by the environment (Banerjee et al. 2016). Sequence-related amplified polymorphic (SRAP), inter-simple sequence repeat (ISSR), simple sequence repeat (SSR), and random amplified polymorphic DNA (RAPD) markers have been used for phylogenetic analyses of mulberry (Banerjee et al. 2016; Sharma et 
69 mulberry accessions and analyze their genetic diversity. The results were consistent with

70 classifications based on morphological characters (Zhao et al. 2005; Zhao et al. 2007). Internal

71 transcribed spacer (ITS) regions (ITS1, 5.8S, and ITS2) are among the most widely used

72 molecular markers in phylogenetic analyses of angiosperms (Alvarez \& Wendel 2003; Baldwin

73 et al. 1996; Li et al. 2011) and are proposed to be core barcodes for seed plants (Li et al. 2011).

74 These markers have also been used in phylogenetic analyses of mulberry. Based on ITS

75 sequences and trnL-trnF sequences data of 13 mulberry accessions and Broussonetia papyrifera,

76 Zhao divided Morus into five major clades, and identified Morus as a monophyletic group (Zhao

77 et al. 2005). Nepal and Ferguson recognized 13 species of Morus, but phylogenetic analyses of

ITS sequences and trnL-trnF sequences determined that this genus is not monophyletic, compared with 12 species in another genus (Trophis, Bagassa, Milicia, Sorocea, Streblus, and Artocarpus) in the Moraceae (Nepal \& Ferguson 2012). Recently, the genus Morus was redefined to contain eight species based on comprehensive analyses of ITS sequences from 43 mulberry accessions and one outgroup (B. papyrifera), and the phylogenetic relationships among clades were determined (Zeng et al. 2015). Most of the mulberry accessions collected in China were grouped into the largest clade B, even though there were many morphological polymorphisms and 12 supposed species among the mulberry accessions in clade B (Zeng et al. 2015). Because the sequence similarity of ITS sequences in clade B was very high, it was difficult to redefine the classification and investigate the evolutionary patterns among them.

Generally, ITS regions are considered to be homologous in an individual as a result of concerted 
evolution (Ghosh et al. 2017; Sang et al. 1995; Wendel et al. 1995), and intra-individual polymorphisms in ITS regions have been regarded as exceptions in many plant groups (Mayol \& Rossello 2001). As one type of ITS polymorphism, ITS pseudogenes were first found in Zea mays (Buckler \& Holtsford 1996) and then in various other plants (Fan et al. 2014; Xiao et al. 2010). The ITS pseudogenes are easily distinguished by their GC content, minimum free energy of secondary structure, the presence of conserved motifs, substitution rates, phylogenetic positions, and copy numbers (Bailey et al. 2003; Queiroz et al. 2011). It has been proposed that some ITS pseudogenes are inherited from the maternal progenitor, which is helpful for understanding the evolutionary history of a species (Hughes et al. 2002). Putative ITS pseudogenes show higher sequence diversities than functional ITS sequences, and so they may provide better resolution and more information in phylogenetic analyses (Xu et al. 2017). In any case, ITS pseudogenes should be included in phylogenetic analyses (Bailey et al. 2003).

In preliminary analyses, we found ITS pseudogene sequences in mulberry, and speculated that they may provide new insights into mulberry evolution. Therefore, we conducted deep sequencing of ITS sequences from 33 mulberry accessions (seven species). We constructed phylogenetic trees using ITS sequences and the chloroplast DNA (cpDNA) sequences trnL-trnF and $\operatorname{trn} T-\operatorname{trn} L$, and found three connection patterns between ITS sequences and cpDNA sequences. Based on analyses of these patterns, we propose that hybridization has played important roles in the evolution of mulberry. The evolutionary history of mulberry is proposed. The results of this study contribute to our understanding of mulberry evolution. 


\section{Materials \& methods}

\section{Plant materials}

114 We selected 33 mulberry accessions (seven species: M. alba, M. mongolica, M. cathayana, M. wittiorum, M. nigra, M. yunnanensis, and M. notabilis) for this study (see Table 1). Morus

yunnanensis was obtained from the Institute of Sericulture and Apiculture, Yunnan Academy of

Agricultural Sciences, Mengzi, Yunnan province, China. The other mulberry accessions were

obtained from the Mulberry Germplasm Nursery at Southwest University, China (He et al. 2013;

Zeng et al. 2015), where they are preserved by propagation through grafting. The relatively

closely related species Artocarpus heterophyllus was selected as the outgroup for the

phylogenetic analyses based on ITS sequence (KT002551) and cpDNA sequences (MG434693).

\section{DNA preparation, sequence selection, and amplification}

Genomic DNA was extracted from all mulberry accessions using the CTAB method (Saghai-

Maroof et al. 1984). Chloroplast DNA was extracted as described by Shi et al (Shi et al. 2012).

Based on the alignment of six whole chloroplast genomes (from M. notabilis (KP939360.1), M.

indica (DQ226511.1), M. cathayana (KU981118.1), M. mongolica (KM491711.2), M. alba var.

multicaulis (KM491711.2), and M. alba var. atropurpurea (KU355276.1)) using VISTA viewer

(http://genome.lbl.gov/vista/index.shtml) (Frazer et al. 2004), we selected the two variable cpDNA sequences, $\operatorname{trn} L-\operatorname{trn} F$ and $\operatorname{trn} T$-trnL, for analyses. The ITS and the $\operatorname{trn} L$-trnF sequences 
132

\section{1}

designed primers to amplify trnT-trnL (F: 5'-TGCGATGCTCTAACCTCT-3'; R: 5'-

TAGCGTCTACCAATTTCG-3'). These sequences were amplified using GoTaq Flexi DNA

Polymerase (Promega Corporation, Madison, WI, USA) according to the manufacturer's instructions. All the polymerase chain reaction (PCR) cycles consisted of initial denaturation of $95^{\circ} \mathrm{C}$ for $5 \mathrm{~min}$; followed by 32 cycles of $95^{\circ} \mathrm{C}$ for $30 \mathrm{~s}$, annealing for $30 \mathrm{~s}, 72{ }^{\circ} \mathrm{C}$ for $1 \mathrm{~min}$; and then final extension for $7 \mathrm{~min}$. The amplified fragments were isolated by electrophoresis on $1 \%(\mathrm{w} / \mathrm{v})$ agarose gels. The purified sequences were cloned into the pMD19-T vector, and then 7-20 positive clones were sequenced for each accession.

Sequence analyses

The cpDNA and ITS sequences were assembled and corrected for PCR errors using Sequencher 4.2 (Gene Codes Corp., Ann Arbor, MI, USA). All the sequences were aligned using Clustal X 1.81 software (Thompson et al. 1997). The boundaries of the ITS1, 5.8S, and ITS2 regions were determined as described elsewhere (Zeng et al. 2015). The GC content and sequence length was calculated using BioEdit (Hall 1999). Three angiosperm conserved motifs (motif 1:

GAATTGCAGAATCC, motif 2: TTTGAACGCA, motif 3: CGATGAAGAACGTAGC) were detected by BioEdit (Kerbs et al. 2017; Yakimowski \& Rieseberg 2014). The minimum free energy of the secondary structure was predicted using Mfold

(http://www.bioinfo.rpi.edu/applications/mfold) (Zuker 2003). 
153 Phylogenetic analyses of ITS sequences and cpDNA sequences were conducted using maximum154 likelihood (M-L) and Bayesian inference (BI) methods. The first phylogenetic analysis was 155 conducted using the ITS sequences of the 33 mulberry accessions determined in this study and 156 those from another nine species (M. mesozygia (HM747171), M. insignis (HM747169), M. 157 serrata (HM747176), M. rubra (HQ144180), M. celtidifolia (HM747168), M. macroura 158 (HM747170), M. mongolica (KF784879), M. wittiorum (AY345154), and M. australis 159 (KT002555)) reported previously (Zeng et al. 2015). The best-fit model SYM+G for BI analyses of ITS sequences was selected by the lowest AIC (Akaike Information Criterion) scores in MrModelTest 2.3 (Klaus-J et al. 2004). MrBayes v3.2.6 software was used for BI analyses (Huelsenbeck \& Ronquist 2001). Four Markov chain Monte Carlo (MCMC) chains were run for 2000000 generations, with sampling every 100 generations. The first 5000 trees were discarded as burn-ins and the 50\% majority-rule consensus tree was determined to calculate the posterior probabilities for each node. The standard deviations of split frequencies were checked and the number of minimum generations required for analysis were those with a standard deviation value lower than 0.01 . The M-L phylogenetic trees were constructed using the most suitable Kimura 2parameter model with the lowest BIC (Bayesian Information Criterion) scores in MEGA 7 (Kumar et al. 2016). A discrete gamma distribution was used to model evolutionary rate differences among sites (five categories $(+\mathrm{G}$, parameter $=1.3541))($ Kumar et al. 2016). All characters were equally weighted.

Phylogenetic analyses of cpDNA sequences were performed using sequence matrix data for the 
174

175

176

177

178

179

180

181

182

183

184

$\operatorname{trnL}$-trnF and trnT-trnL regions. The best-fit model (GTR) for BI and M-L analyses for cpDNA sequences was selected by the lowest AIC scores in MrmodelTest 2.3 (Klaus-J et al. 2004). The other parameters for $\mathrm{BI}$ analyses based on cpDNA sequences were the same as the parameters for analyses based on ITS sequences. The M-L phylogenetic tree was constructed in MEGA 7 with 1000 bootstrap replicates (Kumar et al. 2016). All characters were equally weighted.

\section{Results}

\section{Variations among ITS sequences}

Thirty-three mulberry accessions were sequenced and their ITS sequences were analyzed (Table 1). For each accession, 7-20 clones were sequenced (Table 1). Of the 33 mulberry accessions, 19 contained only one type of ITS sequence, with lengths ranging from $611 \mathrm{bp}$ to $631 \mathrm{bp}$. The ITS sequences of M. notabilis, M. yunnanensis, and M. nigra were confirmed to have lengths of 631 bp, $631 \mathrm{bp}$, and $624 \mathrm{bp}$, respectively, as reported previously (Zeng et al. 2015). Of the 19 mulberry accessions with one ITS sequence, 16 had an ITS sequence of $611 \mathrm{bp}$, with differences at only two single nucleotide polymorphism (SNP) sites.

The other 14 mulberry accessions had polymorphic ITS sequences (Table 1). There were two types of ITS sequences: short ITS sequences (ITS- $\alpha$ ) and long ITS sequences (ITS- $\beta$ ). The ITS sequences of the mulberry accessions Shimiansang, Hanguodabaizhenzhu, Shuisang, and Pisang2 contained two short-type sequences. Sequences with higher and lower copy numbers were designated as ITS- $\alpha 1$ and ITS- $\alpha 2$, respectively. The accession Yun6muben contained two 
195

196

197

198

199

200

201

202

203

204

205

206

207

208

209

210

211

212

213

214

215

long ITS sequences (625 bp and $624 \mathrm{bp}$, designated as ITS- $\beta 1$ and ITS- $\beta 2$, respectively). The

ITS- $\alpha$ sequences in 13 mulberry accessions were identical with a length of $611 \mathrm{bp}$. The length of

ITS- $\beta$ sequences ranged from $624 \mathrm{bp}$ to $626 \mathrm{bp}$. An alignment of the ITS sequences of the

mulberry accessions is shown in Figure 1. We detected the 13-bp InDel reported previously

(Zeng et al. 2015). Further, we found three types of 13-bp sequences among the 33 mulberry

accessions (CGTATACAATGCG, TGTGTGCAATGCG, and CGTACACAATGCG).

Alignment analyses of the ITS sequences revealed six 1-bp and three 2-bp InDels. Other sequence variations were SNPs.

\section{Identification of ITS pseudogenes}

The GC content of the ITS region, the minimum free energy of the secondary structure, and conserved motifs in the 5.8S rDNA region were used to identify ITS pseudogenes. As shown in Figure 2 and Table S1, the GC contents of Shuisang- $\alpha 2$, Jianpuzai- $\beta$, and Yun6- $\beta$ were similar to that of ITS- $\alpha 1 / \alpha$. The GC content of Yun6muben- $\beta 2$ was lower than that of ITS- $\beta 1$. The GC contents of ITS- $\alpha 2 / \beta 2$ sequences in the remaining mulberry accessions were lower than those of ITS- $\alpha 1 / \beta 1$ sequences in at least one region of ITS1, 5.8S rDNA, and ITS2. The minimum free energy of secondary structure showed a similar pattern to that of GC contents, except that it was lower in Shuisang- $\alpha 2$, Jianpuzai- $\beta$, Yun6- $\beta$, and Yun6muben- $\beta 2$ than in ITS- $\alpha 1 / \alpha$ and Yun6mb$\beta 1$. The sequences of Hanguodabaizhenzhu- $\alpha 2$, Shuisang- $\alpha 2$, Yun7- $\beta$, Jianpuzai- $\beta$, Yun6- $\beta$, and Yun6muben- $\beta 2$ contained all three conserved motifs. Based on the sequence length information, the GC content of the ITS region, the minimum free energy of secondary structure, and 
216

217 (Yun6muben- $\beta 2$, Pisang2- $\alpha 2$, Shimiansang- $\alpha 2$, Shanxitiansang- $\beta$, Xinyizhilai- $\beta$, Lunjiao109- $\beta$, Huosang- $\beta$, Agentingsang- $\beta$, Hanguodabaizhenzhu- $\alpha 2$, Baojing7- $\beta$, and Yun7- $\beta$ ).

219

220

221

222 
237 M. notabilis (Chen et al. 2016a), M. mongolica (Kong \& Yang 2016), M. cathayana (Kong \&

238 Yang 2017), M. alba var. multicaulis (Kong \& Yang 2017), and M. alba var. atropurpurea (Hu et

239 al. 2014) (Fig. S1) and sequence alignment analyses, the $\operatorname{trn} L$-trnF and $\operatorname{trn} T$-trnL regions were

240 selected for the cpDNA phylogenetic analyses. In the 33 mulberry accessions, the length of trnL-

$241 \operatorname{trnF}$ ranged from $913 \mathrm{bp}$ to $931 \mathrm{bp}$, and that of trnT-trnL ranged from $1108 \mathrm{bp}$ to $1150 \mathrm{bp}$. The

242 combined data matrix of $\operatorname{trnL}-\operatorname{trnF}$ and $\operatorname{trn} T$-trnL consisted of 2109 aligned nucleotides (Fig. 4).

244 We constructed M-L and BI phylogenetic trees using the trnL-trnF and trnT-trnL data (Fig. 5 and S3). The topologies of the BI trees were disordered (Fig. S3), and some identical sequences were clustered into different clades. Therefore, the M-L trees were used for further analyses.

247 First, M. notabilis and M. yunnanensis were diverged first among the 33 mulberry accessions.

248 The remaining 31 mulberry accessions were separated into two main clades. Clade I contained

24911 accessions of species M. alba (Banqiao6, Basailuona, Huasang, Huai302, Huosang,

Shanxitiansang, Shimiansang, Sililanka, Xinyizhilai, Yidachimu, and Wupisang), two accessions

of species M.mongolica (Jimengsang and Mengsang) (Fig. 4 and 5, Table 1). Clade II contained

10 accessions of species M. alba (Agentingsang, Gailiang10, Hanguodabaizhenzhu, Jianpuzai,

Leshandahongpi, Lunjiao109, Shuisang, Taiwanchaochangguo, Xinjiaposijiguosang, and 
258 Taiwanchaochangguo, and Xinjiaposijiguosang) formed a subclade, and M. nigra was separated

259 from the other mulberry accessions on a long branch (Fig. 5).

260

261 Discussion

262 Characterization and mutation pattern of ITS pseudogenes

263 ITS pseudogenes have been detected in many plants (Fan et al. 2014; Won \& Renner 2005; Xiao

264 et al. 2010; Xu et al. 2017), and should not be excluded from phylogenetic analyses of ITS

265 sequences (Bailey et al. 2003). However, ITS pseudogenes have evolved at a faster rate than

266 functional ITS sequences, which can cause confusion in phylogenetic analyses (Bailey et al.

267 2003; Fan et al. 2014). Thus, comprehensive analyses of ITS sequences are required. The ITS

268 pseudogenes can be easily identified based on their GC content, minimum free energy of

secondary structure, presence of conserved motifs, substitution rates, phylogenetic positions, and

copy numbers (Bailey et al. 2003; Queiroz et al. 2011). In the present study, we found ITS

pseudogenes in 11 mulberry accessions, implying that incomplete concerted evolution is

occurring in mulberry. Compared with functional ITS sequences, the ITS pseudogenes of

mulberry showed higher diversity in the whole ITS region (ITS1, 5.8S rDNA, and ITS2), as

found in other studies (Fan et al. 2014; Xiao et al. 2010). Both the pseudogenes and functional

ITS sequences were a mixture of short- and long-type sequences. Recombination was detected

by the RDP4 program (Martin et al. 2015). The results showed that the short-type pseudogene sequences Shimiansang- $\alpha 2$ and Hanguodabaizhenzhu- $\alpha 2$ have recombined with putative longtype ITS sequences, representing one pathway of concerted evolution of ITS sequences. 


\section{Phylogenetic analyses and evolution of ITS}

281

282

We conducted phylogenetic analyses of ITS functional sequences and pseudogenes. The main topologies of the phylogenetic trees were the same as that reported elsewhere (Zeng et al. 2015).

In the present study, ITS pseudogenes were identified and separated from the functional ITS clades (Fig. 3) (Bailey et al. 2003). Clade 3 is a newly identified clade with long-type ITS sequences compared with the previous study (Zeng et al. 2015). Based on the sequence similarities and sequence length of the ITS region, the long-type ITS sequences in clade 3 may have closer relationships with the sequences in clades A, C, and D (Fig. 1).

In our analyses, most of the sequences were clustered in clade 1 and clade 2 , with only three sequences clustered into clade 3 (Fig. 3). We detected inconsistencies between traditional and molecular classifications in this study. Twenty-nine mulberry accessions with morphological polymorphisms clustered together with a low phylogenetic resolution and had similar or identical ITS sequences. For example, Yun6muben and Yun7muben showed similar fruit, leaf, bud, and internode morphologies, indicating that both should be classified as M. wittiorum. However, Yun6muben had long-type ITS sequences that clustered in clade 3, while Yun7muben had shorttype ITS sequences that clustered in clade 2. As the morphological characteristics were very complex, no details are discussed here. These phenomena are indicative of concerted evolution or introgression of the ITS region, and of hybridization (Bailey et al. 2003; Xu et al. 2017). Thus, the classification of mulberry is very complex, and there is considerable confusion at present. 
300

301

302

303

304

305

306

307

308

309

310

311

312

313

314

315

316

317

318

319

320

Systematic classification of mulberry resources should be based on molecular markers, morphology, chromosome number, genome data, and other characteristics.

Intra-individual polymorphisms in the ITS region have been detected in many plants and

pseudogene sequences have been included in phylogenetic analyses (Mayol \& Rossello 2001;

Xiao et al. 2010; Zheng et al. 2008). Bidirectional, unidirectional, or different rates of evolution

have been detected from analyses of ITS sequences (Wendel et al. 1995; Xu et al. 2017; Zheng et

al. 2008). In this study, we detected multiple functional ITS copies, putative ITS pseudogenes, or

recombinants of ITS pseudogenes in the same individual. The successive evolution patterns of

ITS sequences are indicative of concerted evolution (Fig. 1 and 3). There are several lines of

evidence for the concerted evolution of ITS sequences. First, clades A, C, D, and 3 had long-type

and ancient ITS sequences. Second, Y6 was identified as the progeny of Yun6muben through

natural pollination. Yun6- $\beta$ showed one SNP variation compared with Yun6muben- $\beta 1$,

suggesting that concerted evolution started in the F1 generation. Third, Hanguodabaizhenzhu- $\alpha 2$

and Shimiansang- $\alpha 2$ were found to be recombined from long-type ITS sequences, and other ITS

pseudogenes showed more variations. Fourth, Shuisang- $\alpha 2$ had only one SNP variation

compared with ITS sequences in clade 1 and clade 2 , implying that concerted evolution is

continuing or has completed only recently. Finally, most functional ITS copies were short-type

sequences. In conclusion, the evolutionary process of ITS, as indicated by our data, is that long-

type ITS sequences are undergoing concerted evolution to form short-type ITS sequences. 
321

322

323

324

325

326

327

328

329

330

331

332

333

334

335

336

337

338

339

340

341

Hybridization is an important evolutionary mechanism in plants, especially in flowering plants

(Kerbs et al. 2017; Soltis \& Soltis 2009; Yakimowski \& Rieseberg 2014). Hybridization can also help to explain the concerted evolution of ITS sequences (Xu et al. 2017). We detected three hybridized mulberry accessions (Jianpuzai, Shuisang, and Yun6), which contained two functional ITS sequences. Hybridization between M. rubra (A clade) and M. alba (B clade) has been detected in previous studies (Burgess \& Husband 2004; Burgess et al. 2008). It has been suggested that hybridization between $M$. rubra and M. alba caused the local decline of $M$. rubra (Burgess et al. 2005). This may indicate that M. alba has higher ecological potential. The continuous back-cross hybridization of $M$. rubra with $M$. alba may have led to the disappearance of ITS sequences from M. rubra, or the concerted evolution to form short-type sequences. Like other plants, local Morus species face the risk of extinction (Burgess \& Husband 2004; Burgess et al. 2008; Ellstrand \& Schierenbeck 2000; Wolf et al. 2001).

Polyploidy is another important evolutionary mechanism that is known to affect the concerted evolution of ITS sequences in plants (Ainouche et al. 2004). The ITS sequences can retain their subgenomic sequences or become homogenized through concerted evolution (Wendel et al. 1995). Existing mulberry species show various polyploidy levels, with 14, 28, 35, 42, 49, 56, 84, 112, 126, or 308 chromosomes (Xuan et al. 2017; Zeng et al. 2015). Multiple nucleolus organizing region (NOR) loci have also contributed to the concerted evolution of ITS sequences. Two pairs of NOR loci have been reported for M. notabilis (Xuan et al. 2017). The other mulberry accessions contained at least two pairs of NOR loci (data not shown). Mulberry is often 
342 cultivated by grafting or propagated from cuttings, resulting in a long generation time. This may

343 be another factor affecting the concerted evolution of ITS sequences. In summary, hybridization,

344 polyploidy, multiple NOR loci, and long generation times have all contributed to the concerted

345 evolution of ITS sequences in mulberry.

346

347 Phylogenetic analyses of cpDNA sequences

348 Certain cpDNA sequences are widely used molecular markers in phylogenetic analyses (Nepal \&

349 Ferguson 2012; Xu et al. 2012). With the development of universal primers for cpDNA and

350

351

352

353

354

355

356

357

358

359

360

361

chloroplast genome sequencing, increasing numbers of studies have been conducted based on cpDNA (Huang et al. 2014; Wu et al. 2014). Another characteristic of cpDNA is that it is maternally inherited. To date, six chloroplast genomes of mulberry have been reported, and they can provide comprehensive information about the evolution of the whole chloroplast genome (Chen et al. 2016a; Hu et al. 2014; Kong \& Yang 2016; Kong \& Yang 2017; Ravi et al. 2007).

Several other molecular markers have been used in phylogenetic studies, with trnL-trnF being the most commonly used (Ayinampudi et al. 2011; Nepal \& Ferguson 2012). In this study, more variable cpDNA regions (trnL-trnF and $\operatorname{trn} T-\operatorname{trn} L)$ were used for phylogenetic analyses to study the concerted evolution of mulberry. The mulberry accessions in clade B were separated into two clades in the phylogenetic tree based on cpDNA sequences. The sequences in clade I were almost identical, sequences in clade II showed more variations and a subclade was clustered (Fig. 4 and 5). Successive evolution pattern of the sequences was shown in Figure 4, suggesting 
362 a closer progenitor among them. Thus, clade II could be treated as a single clade. These results

363 were consistent with those of previous studies in which two clades were proposed based on

364 phylogenetic analyses of TrnL-TrnF (Zhao et al. 2005; Zhao et al. 2007).

365

366

\section{Evolution of mulberry}

Based on the analyses of ITS sequences, the 33 functional ITS sequences in clade B were separated into short-type sequence clades (clade 1 and clade 2) and a long-type sequence clade (clade 3). Two pseudogroups were also divided in the ITS phylogenetic tree. These findings suggest that there is a shallow level of phylogeny among 29 mulberry accessions, consistent with the two clades detected in the cpDNA phylogenetic tree (Bailey et al. 2003). Thus, clade B contains two species.

On the basis of a study of the genus Leucaena, it was proposed that some ITS pseudogene sequences are inherited from the maternal progenitor (Hughes et al. 2002). In this study, we detected three connection patterns between ITS pseudogene sequences and cpDNA sequences

377 (Fig. 1 and 4). Connection pattern (1) was between pseudogroup 2 and clade I, and was detected in Shanxitiansang, Xinyizhilai, Huosang, and Shimiansang. In this pattern, both the ITS pseudogene sequences and cpDNA sequences showed high sequence similarities. Connection pattern (2) was between pseudogroup 1 and clade II, and was detected in Yun7, Yun6muben, displayed sequence variations, but the cpDNA sequences did not. Connection pattern (3) was 
383

384

385

386

387

388

389

390

391

392

393

394

395

396

397

398

399

400

401

402 403

between pseudogroup 2 and clade II, and was detected in Hanguodabaizhenzhu, Lunjiao109, and

Agentingsang. In these three patterns, the ITS pseudogene sequences have connections with genetic information from the female progenitor. This provides more clues to trace the evolution process of ITS sequences and the Morus genus.

The three connection patterns and the incongruent distribution patterns between the cpDNA and the ITS phylogenetic trees imply that hybridization has contributed to the evolution of mulberry. Based on the discussion above, a simple evolutionary pattern is proposed for mulberry (Fig. 6). First, mulberry with short-type ITS sequences ( $\left.{ }^{\mathrm{a} A A}\right)$ existed at a certain time and showed stronger ecological potential. Those resources then hybridized with mulberry with long-type sequences ( $\left.{ }^{b} \mathrm{BB}\right)$. The ITS sequences gradually became short-type sequences through concerted evolution or hybridization. Finally, long-type ITS sequences (B) were left as pseudogene sequences $(B)$ in the offspring, and most mulberry had short-type functional ITS sequences $\left({ }^{\mathrm{a} A \mathrm{~A}}\right.$, ${ }^{\mathrm{a}} \mathrm{AA}^{B}$, and $\left.{ }^{\mathrm{b}} \mathrm{AA}^{B}\right)$. Thus, mulberry with long-type ITS sequences $\left({ }^{\mathrm{b}} \mathrm{BB}\right)$ are the original mulberry resources, and are being polluted by those with short-type ITS sequences during evolution. The actual evolutionary process of mulberry will be more complex than this evolutionary pattern, and dominated by hybridization.

In the phylogenetic trees constructed from ITS and cpDNA sequences (Fig. 3 and 5), all the clades contained accessions with different ploidy levels, and there were cross-links between the clades in different trees (e.g. Huai302 $(2 n=12 x=84)$ belonged to clade 2 and clade I). These 
404 results indicate that hybridization and polyploidy have played important roles in the evolution of

405 mulberry, even in the accessions with higher ploidy levels. Clade 2 of the phylogenetic tree

406 constructed from ITS sequences contained mulberry accessions with different high ploidy levels,

407 identical ITS sequences, and successively evolved cpDNA sequences, implying that concerted

408 evolution has played a key role in the evolution of this clade. In conclusion, polyploidy,

409 hybridization, and concerted evolution have all played important roles in the evolution of ITS

410 sequences in mulberry.

412 Conclusions

Based on phylogenetic analyses of ITS sequences and cpDNA sequences, clade B was separated into two species. We found ITS pseudogenes in mulberry, and detected a concerted evolutionary

process in the direction of short-type ITS sequences. We detected three connection patterns between ITS pseudogene sequences and cpDNA sequences, suggesting that the ITS pseudogene sequences connect with genetic information from the female progenitor. Combining the three connection patterns and incongruent distribution patterns between phylogenetic trees constructed from cpDNA and ITS sequences, hybridization is recent or still occurring, and has played important roles in mulberry evolution. The proposed evolutionary pattern, in which hybridization is a key feature, helps us to understand the evolutionary history of mulberry and highlights the importance of conserving local resources. Finally, polyploidy, hybridization, and concerted evolution have all played roles in the evolution of ITS sequences in mulberry. 


\section{Acknowledgments}

Not applicable.

\section{References}

Ainouche ML, Baumel A, Salmon A, and Yannic G. 2004. Hybridization, polyploidy and speciation in Spartina (Poaceae). New Phytologist 161:165-172. DOI: 10.1046/j.1469-8137.2003.00926.x.

Alvarez I, and Wendel JF. 2003. Ribosomal ITS sequences and plant phylogenetic inference. Molecular Phylogenetics and Evolution 29:417-434. DOI: 10.1016/S1055-7903(03)00208-2.

Ayinampudi SR, Wang YH, Avula B, Smillie TJ, and Khan IA. 2011. Quantitative Analysis of Oxyresveratrol in Different Plant Parts of Morus Species and Related Genera by HPTLC and HPLC. Jpc-Journal of Planar Chromatography-Modern Tlc 24:125-129. DOI: 10.1556/Jpc.24.2011.2.8.

Bailey CD, Carr TG, Harris SA, and Hughes CE. 2003. Characterization of angiosperm nrDNA polymorphism, paralogy, and pseudogenes. Molecular Phylogenetics and Evolution 29:435-455. DOI: 10.1016/j.ympev.2003.08.021.

Baldwin BG, Sanderson MJ, Porter JM, Wojciechowski MF, Campbell CS, and Donoghue MJ. 1996. The ITS region of nuclear ribosomal DNA: A valuable source of evidence on angiosperm phylogeny (vol 82, pg 247, 1995). Annals of the Missouri Botanical Garden 83:151-151. Doi 10.2307/2399971.

Banerjee R, Chattopadhyay S, and Saha AK. 2016. Genetic Diversity and Relationship of Mulberry Genotypes Revealed by RAPD and ISSR Markers. Journal of Crop Improvement 30:478-492. DOI: 10.1080/15427528.2016.1177803.

Botton A, Barcaccia G, Cappellozza S, Da Tos R, Bonghi C, and Ramina A. 2005. DNA fingerprinting sheds light on the origin of introduced mulberry (Morus spp.) accessions in Italy. Genetic Resources and Crop Evolution 52:181-192. DOI: 10.1007/s10722-003-4429-x.

Buckler ES, and Holtsford TP. 1996. Zea ribosomal repeat evolution and substitution patterns. Molecular Biology and Evolution 13:623-632. DOI: 10.1093/oxfordjournals.molbev.a025622.

Bureau LÉ. 1873. Moraceae. In: DeCandolle AP, editor. Prodromus systematis naturalis regni vegetabilis. Paris, France: Tuettel and Wurtz. pp. 211-288.

Burgess KS, and Husband BC. 2004. Maternal and paternal contributions to the fitness of hybrids between red and white mulberry (Morus Moraceae). American Journal of Botany 91:1802-1808. DOI: 10.3732/ajb.91.11.1802.

Burgess KS, Morgan M, Deverno L, and Husband BC. 2005. Asymmetrical introgression between two Morus species (M-alba, M-rubra) that differ in abundance. Molecular Ecology 14:3471-3483. DOI: 10.1111/j.1365294X.2005.02670.x.

Burgess KS, Morgan M, and Husband BC. 2008. Interspecific seed discounting and the fertility cost of hybridization in an endangered species. New Phytologist 177:276-283. DOI: 10.1111/j.1469-8137.2007.02244.x.

Chen C, Zhou W, Huang Y, and Wang ZZ. 2016a. The complete chloroplast genome sequence of the mulberry Morus notabilis (Moreae). Mitochondrial DNA Part A 27:2856-2857. DOI:10.3109/19401736.2015.1053127.

Chen H, Pu JS, Liu D, Yu WS, Shao YY, Yang GW, Xiang ZH, and He NJ. 2016b. Anti-Inflammatory and 
464

465

466

467

468

469

470

471

472

473

474

475

476

477

478

479

480

481

482

483

484

485

486

487

488

489

490

491

492

493

494

495

496

497

498

499

500

501

502

503

504

Antinociceptive Properties of Flavonoids from the Fruits of Black Mulberry (Morus nigra L.). Plos One 11. ARTN e0153080. DOI: 10.1371/journal.pone.0153080.

Ellstrand NC, and Schierenbeck KA. 2000. Hybridization as a stimulus for the evolution of invasiveness in plants? Proceedings of the National Academy of Sciences of the United States of America 97:7043-7050. DOI: 10.1073/pnas.97.13.7043.

Fan X, Liu J, Sha LN, Sun GL, Hu ZQ, Zeng J, Kang HY, Zhang HQ, Wang Y, Wang XL, Zhang L, Ding CB, Yang RW, Zheng YL, and Zhou YH. 2014. Evolutionary pattern of rDNA following polyploidy in Leymus (Triticeae: Poaceae). Molecular Phylogenetics and Evolution 77:296-306. DOI: 10.1016/j.ympev.2014.04.016.

Frazer KA, Pachter L, Poliakov A, Rubin EM, and Dubchak I. 2004. VISTA: computational tools for comparative genomics. Nucleic Acids Research 32:W273-W279. DOI: 10.1093/nar/gkh458.

Ghosh JS, Bhattacharya S, and Pal A. 2017. Molecular phylogeny of 21 tropical bamboo species reconstructed by integrating non-coding internal transcribed spacer (ITS1 and 2) sequences and their consensus secondary structure. Genetica 145:319-333. DOI: 10.1007/s10709-017-9967-9.

Hall TA. 1999. BioEdit: a user-friendly biological sequence alignment editor and analysis program for Windows 95/98/NT. 41.

He NJ, Zhang C, Qi XW, Zhao SC, Tao Y, Yang GJ, Lee TH, Wang XY, Cai QL, Li D, Lu MZ, Liao ST, Luo GQ, He RJ, Tan X, Xu YM, Li T, Zhao AC, Jia L, Fu Q, Zeng QW, Gao C, Ma B, Liang JB, Wang XL, Shang JZ, Song PH, Wu HY, Fan L, Wang Q, Shuai Q, Zhu JJ, Wei CJ, Zhu-Salzman K, Jin DC, Wang JP, Liu T, Yu MD, Tang CM, Wang ZJ, Dai FW, Chen JF, Liu Y, Zhao ST, Lin TB, Zhang SG, Wang JY, Wang J, Yang HM, Yang GW, Wang J, Paterson AH, Xia QY, Ji DF, and Xiang ZH. 2013. Draft genome sequence of the mulberry tree Morus notabilis. Nature Communications 4. Artn 2445. DOI: 10.1038/Ncomms3445.

Hu DC, Zhang P, Sun YL, Zhang SM, Wang ZH, and Chen CJ. 2014. Genetic relationship in mulberry (Morus L.) inferred through PCR-RFLP and trnD-trnT sequence data of chloroplast DNA. Biotechnology \& Biotechnological Equipment 28:425-430. DOI: 10.1080/13102818.2014.928980.

Huang H, Shi C, Liu Y, Mao SY, and Gao LZ. 2014. Thirteen Camellia chloroplast genome sequences determined by high-throughput sequencing: genome structure and phylogenetic relationships. Bmc Evolutionary Biology 14. Artn 151. DOI: 10.1186/1471-2148-14-151.

Huelsenbeck JP, and Ronquist F. 2001. MRBAYES: Bayesian inference of phylogenetic trees. Bioinformatics 17:754755. DOI: 10.1093/bioinformatics/17.8.754.

Hughes CE, Bailey CD, and Harris SA. 2002. Divergent and reticulate species relationships in Leucaena (Fabaceae) inferred from multiple data sources: Insights into polyploid origins and nrdna polymorphism. American Journal of Botany 89:1057-1073. DOI: 10.3732/ajb.89.7.1057.

Kerbs B, Ressler J, Kelly JK, Mort ME, Santos-Guerra A, Gibson MJS, Caujape-Castells J, and Crawford DJ. 2017. The potential role of hybridization in diversification and speciation in an insular plant lineage: insights from synthetic interspecific hybrids. Aob Plants 9. ARTN plx043. DOI:10.1093/aobpla/plx043.

Klaus-J, uuml, Ouml R, and rster. 2004. Bayesian phylogenetic analysis of combined data. Systematic Biology 53:4767.

Koidzumi G. 1917. Taxonomical discussion on Morus plants. Bulletin of Sericultural Experimental Station. 3: 1-62.

Kong WQ, and Yang JH. 2016. The complete chloroplast genome sequence of Morus mongolica and a comparative analysis within the Fabidae clade. Current Genetics 62:165-172. DOI: 10.1007/s00294-015-0507-9.

Peer) reviewing PDF | (2019:06:38248:2:0:NEW 19 Oct 2019) 
Kong WQ, and Yang JH. 2017. The complete chloroplast genome sequence of Morus cathayana and Morus multicaulis, and comparative analysis within genus Morus L. Peerj 5. Artn E3037. DOI: 10.7717/Peerj.3037.

Kumar S, Stecher G, and Tamura K. 2016. MEGA7: Molecular Evolutionary Genetics Analysis Version 7.0 for Bigger Datasets. Molecular Biology and Evolution 33:1870-1874. DOI: 10.1093/molbev/msw054.

Li DZ, Gao LM, Li HT, Wang H, Ge XJ, Liu JQ, Chen ZD, Zhou SL, Chen SL, Yang JB, Fu CX, Zeng CX, Yan HF, Zhu YJ, Sun YS, Chen SY, Zhao L, Wang K, Yang T, Duan GW, and Grp CPB. 2011. Comparative analysis of a large dataset indicates that internal transcribed spacer (ITS) should be incorporated into the core barcode for seed plants. Proceedings of the National Academy of Sciences of the United States of America 108:1964119646. DOI: 10.1073/pnas.1104551108.

Linnaeus C. 1753. Morus. Species Plantarum. Stockholm Impensis Laurentii Salvii. pp. 968.

Martin DP, Murrell B, Golden M, Khoosal A, and Muhire B. 2015. RDP4: Detection and analysis of recombination patterns in virus genomes. Virus Evolution 1. UNSP vev003. DOI: 10.1093/ve/vev003.

Mayol M, and Rossello JA. 2001. Why nuclear ribosomal DNA spacers (ITS) tell different stories in Quercus. Molecular Phylogenetics and Evolution 19:167-176. DOI: 10.1006/mpev.2001.0934.

Nepal MP, and Ferguson CJ. 2012. Phylogenetics of Morus (Moraceae) Inferred from ITS and trnL-trnF Sequence Data. Systematic Botany 37:442-450. DOI: 10.1600/036364412x635485.

Priya S. 2012. Medicinal values of mulberry. J Pharm Res 5:3588-3596.

Queiroz CD, Batista FRD, and de Oliveira LO. 2011. Evolution of the 5.8S nrDNA gene and internal transcribed spacers in Carapichea ipecacuanha (Rubiaceae) within a phylogeographic context. Molecular Phylogenetics and Evolution 59:293-302. DOI: 10.1016/j.ympev.2011.01.013.

Ravi V, Khurana JP, Tyagi AK, and Khurana P. 2007. The chloroplast genome of mulberry: complete nucleotide sequence, gene organization and comparative analysis. Tree Genetics \& Genomes 3:49-59. DOI: 10.1007/s11295-006-0051-3.

Sánchez MD. 2002. World Distribution and Utilization of Mulberry, Potential for Animal Feeding. Fao Animal Production \& Health Paper.

Saghai-Maroof MA, Soliman KM, Jorgensen RA, and Allard RW. 1984. Ribosomal DNA spacer-length polymorphisms in barley: mendelian inheritance, chromosomal location, and population dynamics. Proceedings of the National Academy of Sciences of the United States of America 81:8014-8018.

Sanchez DM. 2002. World distribution and utilization of mulberry and its potential for animal feeding. Fao Animal Production \& Health Paper.

Sang T, Crawford DJ, and Stuessy TF. 1995. Documentation of Reticulate Evolution in Peonies (Peonia) Using Internal Transcribed Spacer Sequences of Nuclear Ribosomal DNA - Implications for Biogeography and Concerted Evolution. Proceedings of the National Academy of Sciences of the United States of America 92:6813-6817. DOI: 10.1073/pnas.92.15.6813.

Sharma A, Sharma R, and Machii H. 2000. Assessment of genetic diversity in a Morus germplasm collection using fluorescence-based AFLP markers. Theoretical and Applied Genetics 101:1049-1055. DOI: $10.1007 / \mathrm{s} 001220051579$.

Shi C, Hu N, Huang H, Gao J, Zhao YJ, and Gao LZ. 2012. An Improved Chloroplast DNA Extraction Procedure for Whole Plastid Genome Sequencing. PLoS One 7. ARTN e31468. DOI: 10.1371/journal.pone.0031468.

Soltis PS, and Soltis DE. 2009. The Role of Hybridization in Plant Speciation. Annual Review of Plant Biology 60:561- 
588. DOI: 10.1146/annurev.arplant.043008.092039.

Taberlet P, Gielly L, Pautou G, and Bouvet JJPMB. 1991. Universal primers for amplification of three non-coding regions of chloroplast DNA. 17:1105-1109. DOI: 10.1007/bf00037152.

Thompson JD, Gibson TJ, Plewniak F, Jeanmougin F, and Higgins DG. 1997. The CLUSTAL_X windows interface: flexible strategies for multiple sequence alignment aided by quality analysis tools. Nucleic Acids Research 25:4876-4882. DOI: 10.1093/nar/25.24.4876.

Vijayan K, Srivastava PP, and Awasthi AK. 2004. Analysis of phylogenetic relationship among five mulberry (Morus) species using molecular markers. Genome 47:439-448. DOI: 10.1139/G03-147.

Wendel JF, Schnabel A, and Seelanan T. 1995. Bidirectional Interlocus Concerted Evolution Following Allopolyploid Speciation in Cotton (Gossypium). Proceedings of the National Academy of Sciences of the United States of America 92:280-284. DOI: 10.1073/pnas.92.1.280.

Wolf DE, Takebayashi N, and Rieseberg LH. 2001. Predicting the risk of extinction through hybridization. Conservation Biology 15:1039-1053. DOI: 10.1046/j.1523-1739.2001.0150041039.x.

Won H, and Renner SS. 2005. The internal transcribed spacer of nuclear ribosomal DNA in the gymnosperm Gnetum. Molecular Phylogenetics and Evolution 36:581-597. DOI: 10.1016/j.ympev.2005.03.011.

Wu ZH, Gui ST, Quan ZW, Pan L, Wang SZ, Ke WD, Liang DQ, and Ding Y. 2014. A precise chloroplast genome of Nelumbo nucifera (Nelumbonaceae) evaluated with Sanger, Illumina MiSeq, and PacBio RS II sequencing platforms: insight into the plastid evolution of basal eudicots. Bmc Plant Biology 14. ARTN 289. DOI: 10.1186/s12870-014-0289-0.

Xiao LQ, Moller M, and Zhu H. 2010. High nrDNA ITS polymorphism in the ancient extant seed plant Cycas: Incomplete concerted evolution and the origin of pseudogenes. Molecular Phylogenetics and Evolution 55:168-177. DOI: 10.1016/j.ympev.2009.11.020.

$\mathrm{Xu}$ B, Wu N, Gao XF, and Zhang LB. 2012. Analysis of DNA sequences of six chloroplast and nuclear genes suggests incongruence, introgression, and incomplete lineage sorting in the evolution of Lespedeza (Fabaceae). Molecular Phylogenetics and Evolution 62:346-358. DOI: 10.1016/j.ympev.2011.10.007.

Xu B, Zeng XM, Gao XF, Jin DP, and Zhang LB. 2017. ITS non-concerted evolution and rampant hybridization in the legume genus Lespedeza (Fabaceae). Scientific Reports 7. Artn 40057. DOI: 10.1038/Srep40057.

Xuan YH, Li CS, Wu Y, Ma B, Liu RL, and He NJ. 2017. FISH-based mitotic and meiotic diakinesis karyotypes of Morus notabilis reveal a chromosomal fusion-fission cycle between mitotic and meiotic phases. Scientific Reports 7. ARTN 9573. DOI: 10.1038/s41598-017-10079-6.

Yakimowski SB, and Rieseberg LH. 2014. The Role of Homoploid Hybridization in Evolution: A Century of Studies Synthesizing Genetics and Ecology. American Journal of Botany 101:1247-1258. DOI: 10.3732/ajb.1400201.

Zeng QW, Chen HY, Zhang C, Han MJ, Li T, Qi XW, Xiang ZH, and He NJ. 2015. Definition of Eight Mulberry Species in the Genus Morus by Internal Transcribed Spacer-Based Phylogeny. PLoS One 10. ARTN e0135411. DOI: 10.1371/journal.pone.0135411.

Zhao WG, Pan YL, Zhang ZF, Jia SH, Miao XX, and Huang YP. 2005. Phylogeny of the genus Morus (Urticales : Moraceae) inferred from ITS and trnL-F sequences. African Journal of Biotechnology 4:563-569.

Zhao WG, Zhou ZH, Miao XX, Zhang Y, Wang SB, Huang JH, Xiang H, Pan YL, and Huang YP. 2007. A comparison of genetic variation among wild and cultivated Morus Species (Moraceae : Morus) as revealed by ISSR and SSR markers. Biodiversity and Conservation 16:275-290. DOI: 10.1007/s10531-005-6973-5. 
586

587

588

589

590

591

592

593

594

595
Zheng XY, Cai DY, Yao LH, and Teng YW. 2008. Non-concerted ITS evolution, early origin and phylogenetic utility of ITS pseudogenes in Pyrus. Molecular Phylogenetics and Evolution 48:892-903. DOI: 10.1016/j.ympev.2008.05.039.

Zhou ZK, Gilbert MG. 2003. Moraceae. In: Wu ZY, Raven PH, Hong DY, editors. Flora of China. Beijing, China \& Saint Louis, Missouri: Science Press \& Missouri Botanical Garden Press. pp. 22-26.

Zuker M. 2003. Mfold web server for nucleic acid folding and hybridization prediction. Nucleic Acids Research 31:3406-3415. DOI: $10.1093 / \mathrm{nar} / \mathrm{gkg} 595$. 
Figure 1

\section{Distribution of different sites across ITS region.}

ITS sequence of Morus notabilis was used as reference. Colored lines indicate sites with lower ratios: vermilion line $=\mathrm{A}$; bluish green line $=\mathrm{T}$; purple line $=\mathrm{G}$; sky blue line $=\mathrm{C}$; yellow line = gap; black line $=$ whole ITS sequence. ITS pseudogene sequences were marked with $*$. The clade names were come from Figure 3.

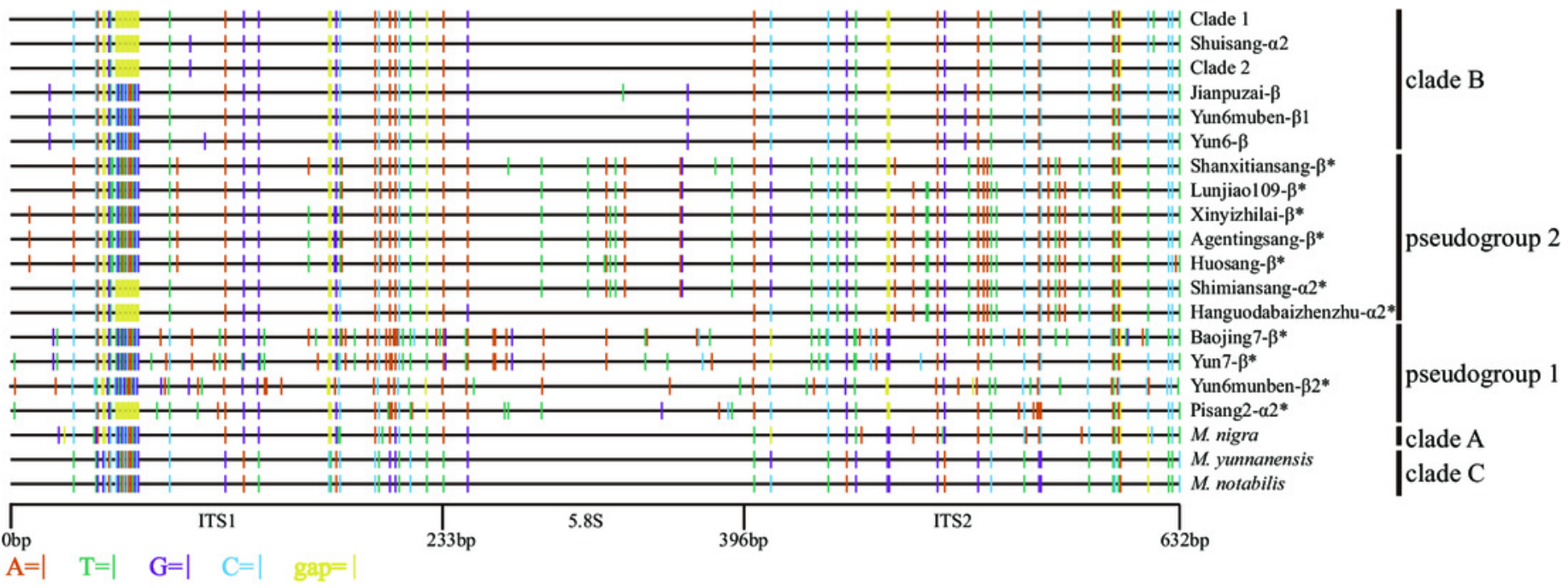


Figure 2

Identification of ITS pseudogenes.

GC content (quadrangle), minimum free energy (triangle), and conserved motifs in 5.8S rDNA were analyzed. Shuisang, Jianpuzai, and Yun6 have two functional ITS sequences and are shown in red.

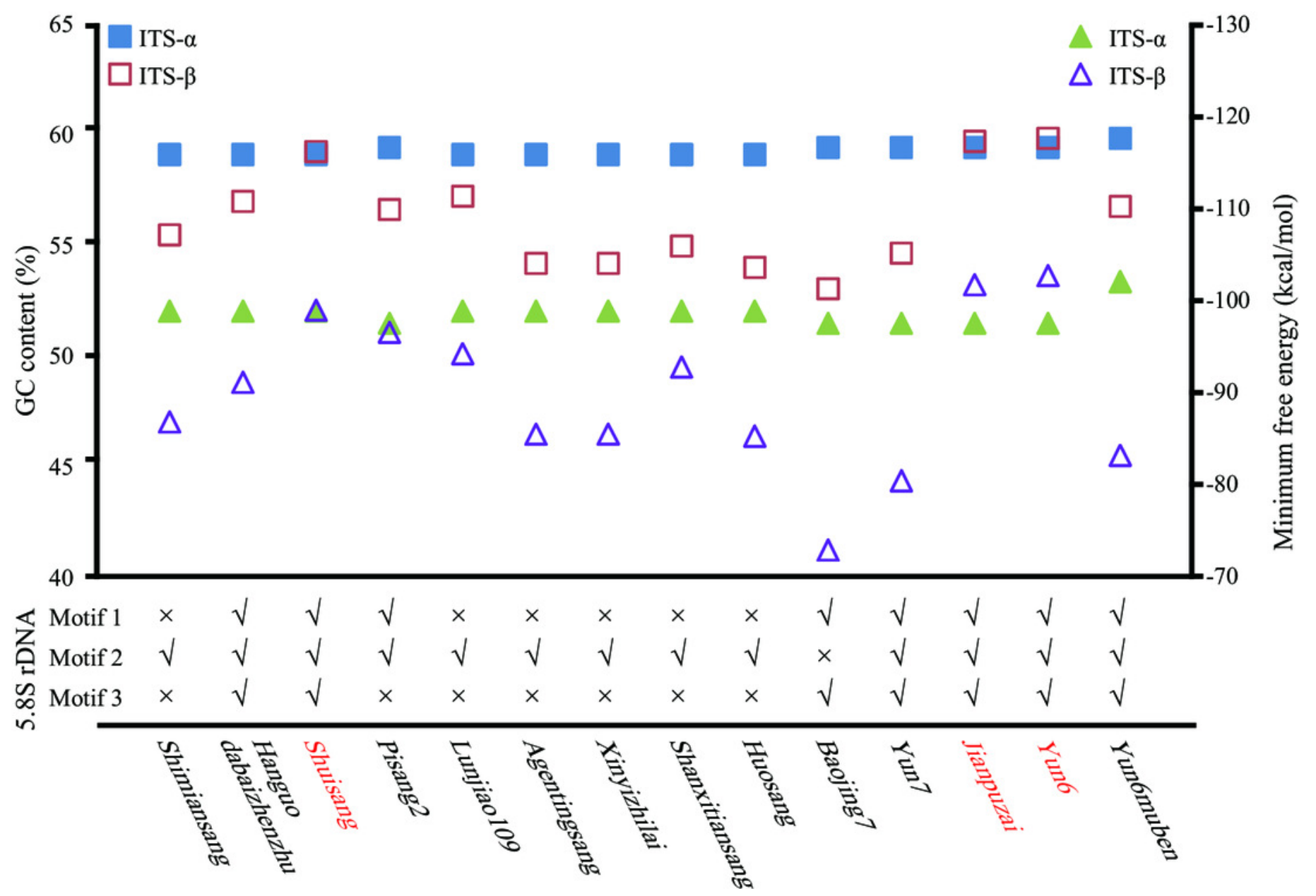




\section{Figure 3}

Maximum-Likelihood phylogenetic tree based on ITS sequences of 33 mulberry accessions.

Phylogenetic tree was constructed using Kimura 2-parameter model in MEGA 7. Clade B was separated into short-type sequence clade (blue clade 1 and sky blue clade 2), long-type sequence clade (vermilion clade 3, bluish green pseudogroup 1, and reddish purple pseudogroup 2). Other clades reported by (Zeng et al. 2015) were shown in black font. ITS pseudogene sequences were marked with *. Bootstrap support values for ML below $50 \%$ are not shown. 


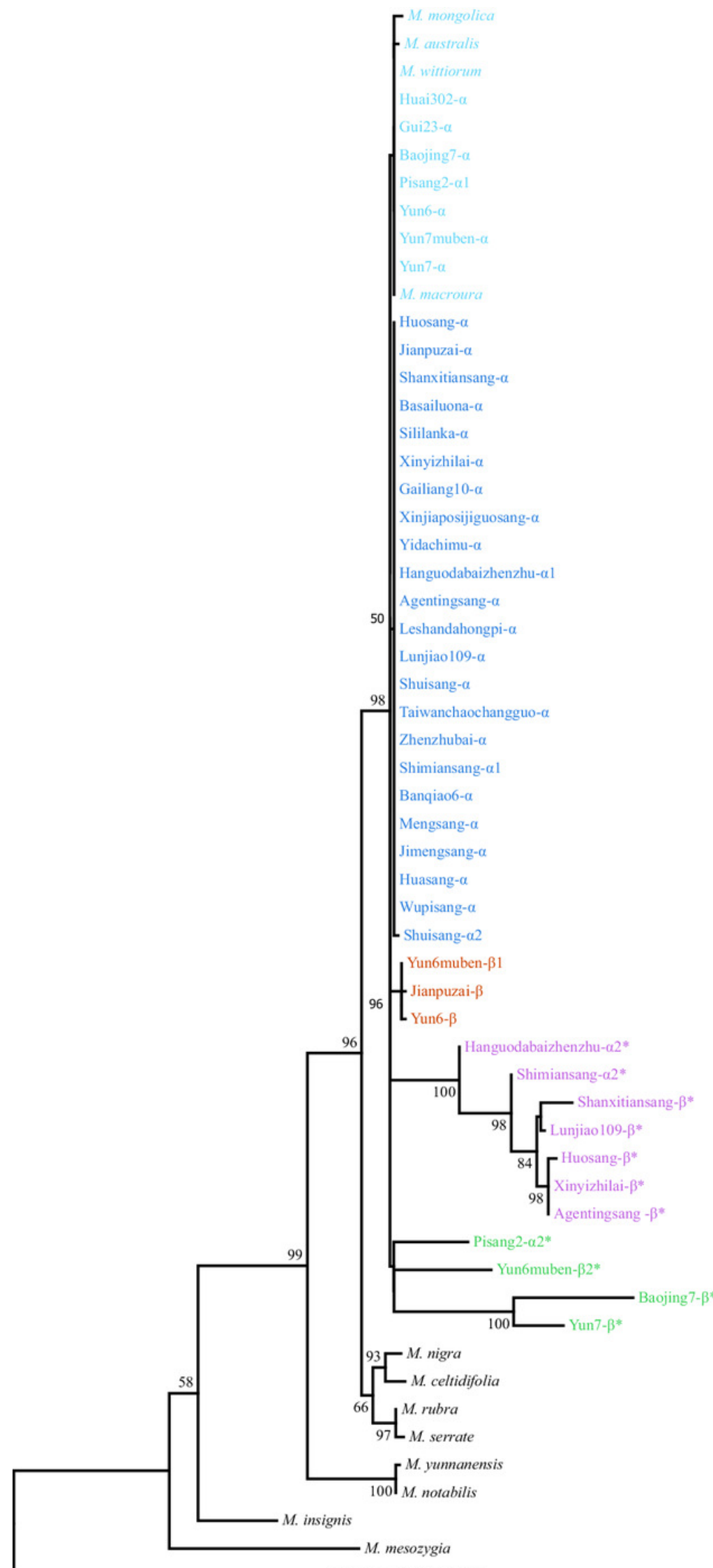

Artocarpus heterophyllus

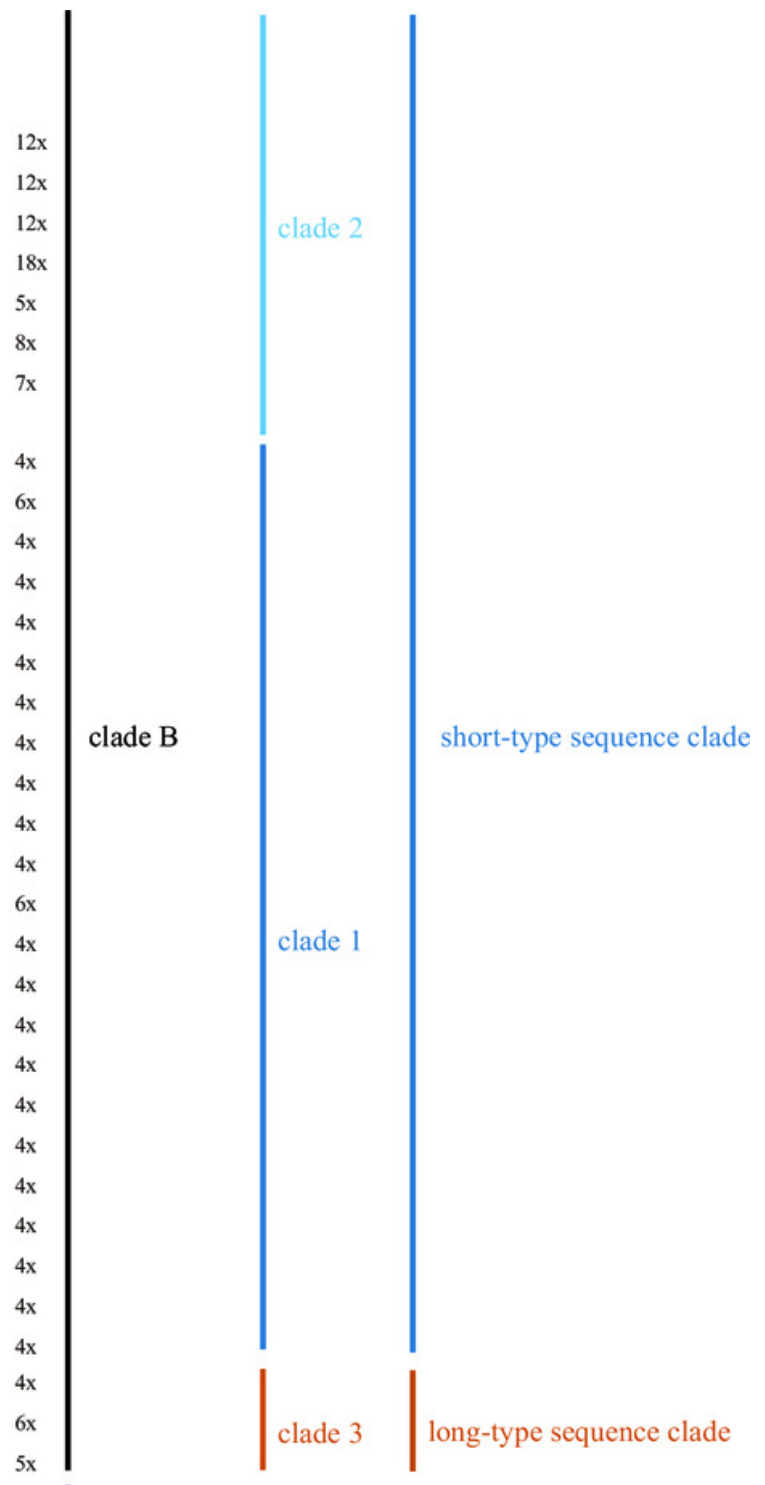

$4 \mathrm{x} \quad$ pseudogroup 2

$4 \mathrm{x}$

$4 \mathrm{x}$

$4 \mathrm{x}$

$18 \mathrm{x}$
$4 \mathrm{x}$

12x pseudogroup 1

$7 \mathrm{x}$

$44 x$

clade A

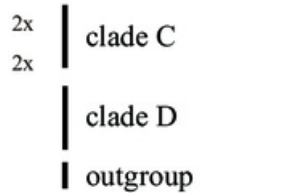

$\stackrel{1}{0.020}$ 
Figure 4

Distribution of different sites across trnL-trnF and trnT-trnL regions.

Sequence of Morus notabilis was used as reference. Colored sites indicate sites with lower ratios: vermilion line $=A$; bluish green line $=T$; purple line $=G$; sky blue line $=\mathrm{C}$; yellow line = gap; black line $=$ whole $\operatorname{trn} L$-trnF and trnT-trnL sequences. The clade names were come from Figure 5.

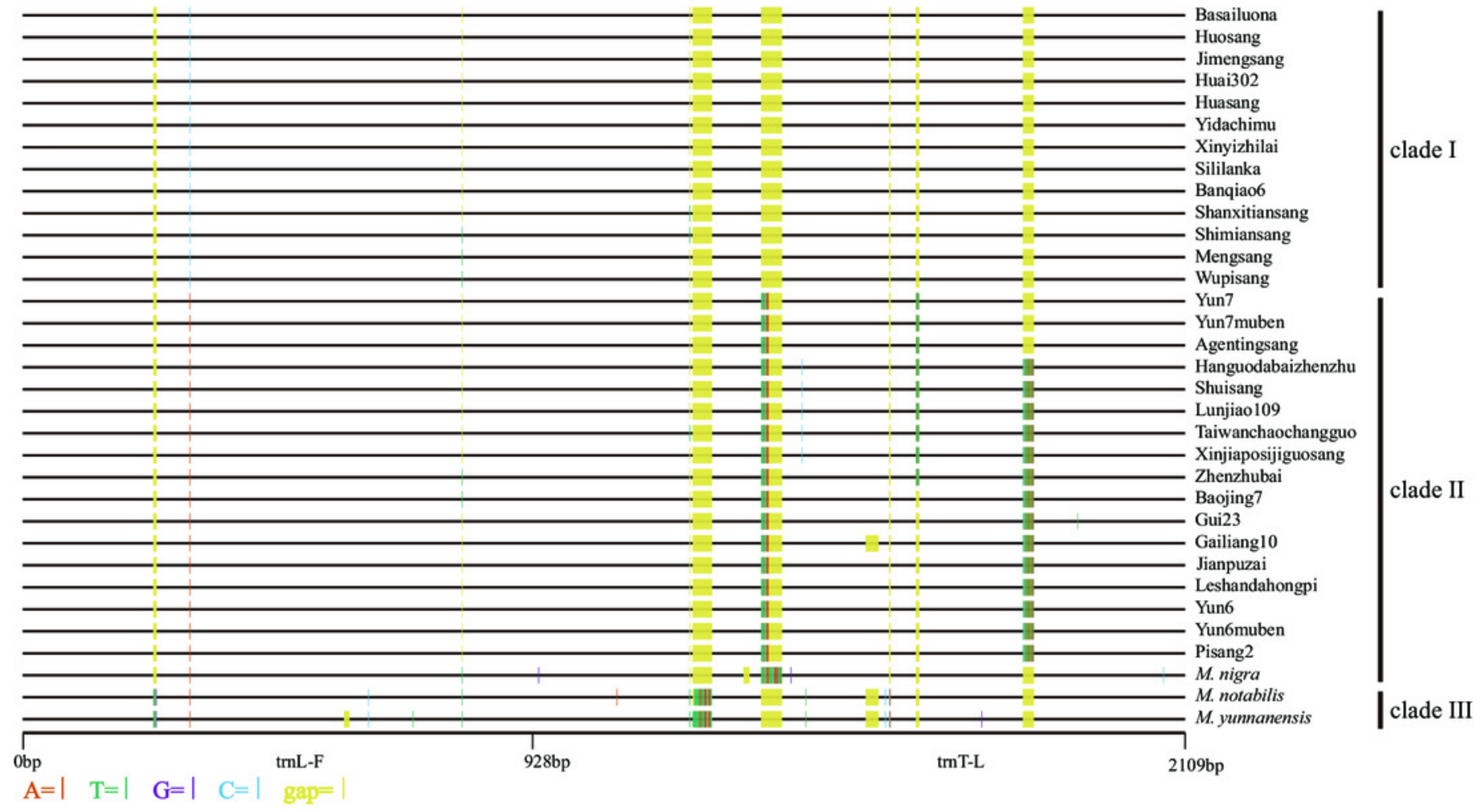


Figure 5

Maximum-likelihood phylogenetic tree based on trnL-trnF and trnT-trnL regions of 33 mulberry accessions.

Phylogenetic tree was constructed using Kimura 3-parameter model in MEGA 7; thirty-three mulberry accessions clustered into clade I, clade II, and clade III . 


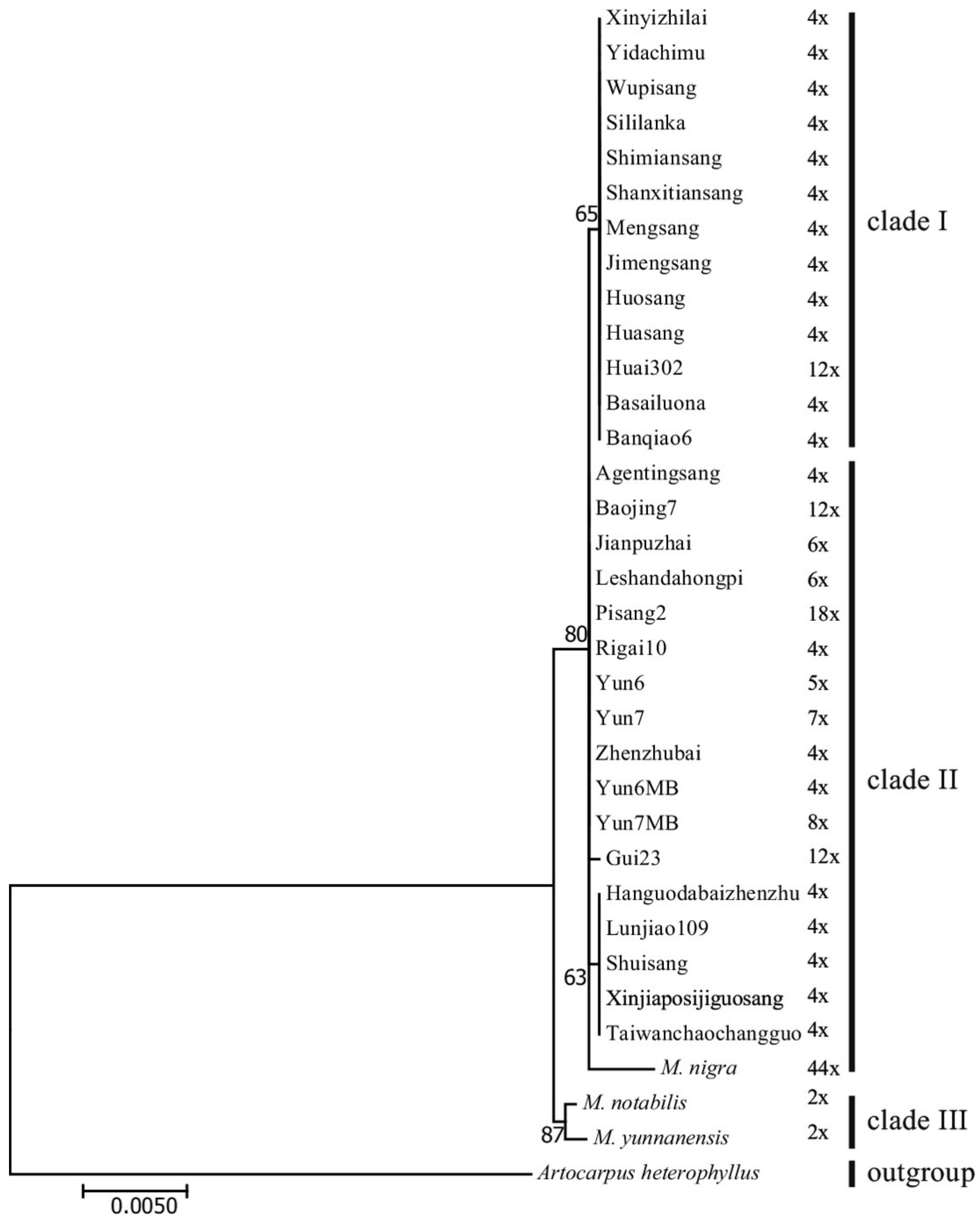




\section{Figure 6}

Simple evolutionary pattern of mulberry.

Mulberry with short-type ( $\left.{ }^{\mathrm{a}} \mathrm{AA}\right)$ and long-type ( $\left.{ }^{\mathrm{b}} \mathrm{BB}\right)$ ITS sequences continuously hybridized with mulberry with short-type ITS sequence mulberry ( $\left.{ }^{\mathrm{A} A A}\right)$. Finally, ${ }^{\mathrm{A} A A}$ mulberry have become most abundant. Mulberry with ${ }^{\mathrm{a}} \mathrm{A} \mathrm{A}^{B}$ and ${ }^{\mathrm{b}} \mathrm{A} \mathrm{A}^{B}$ contain ITS pseudogene sequences ( $B$ ) from different female progenitors. ${ }^{\mathrm{b} B B}$ represents mulberry with long-type ITS sequences. Dotted arrow represents continuous hybridization process. 


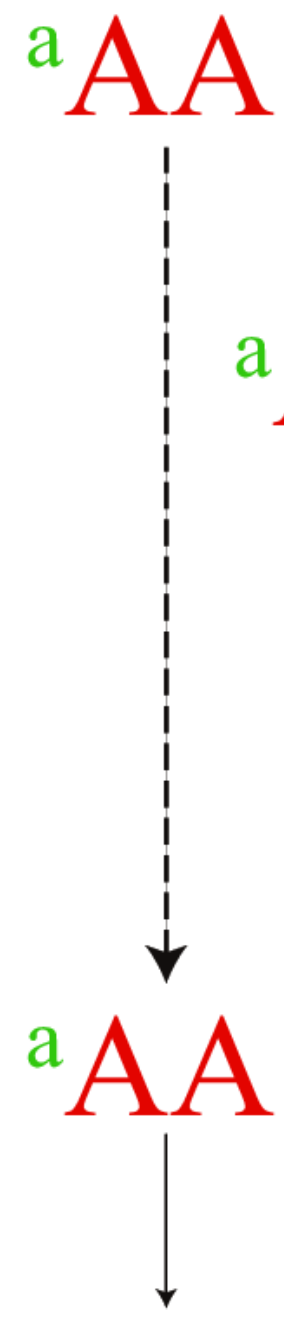

Huasang

Clade 1, 2; clade I

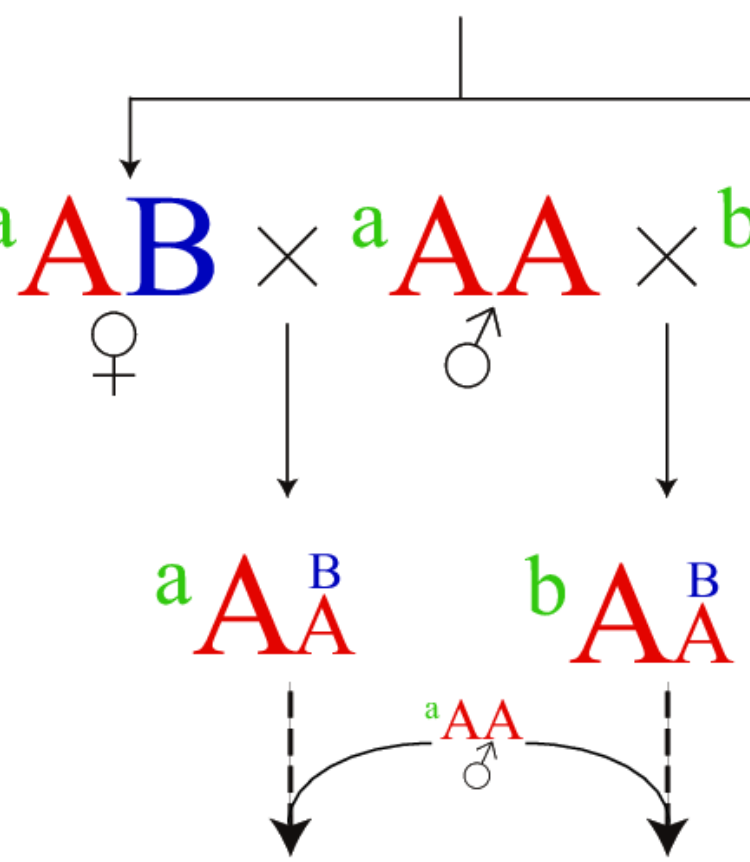

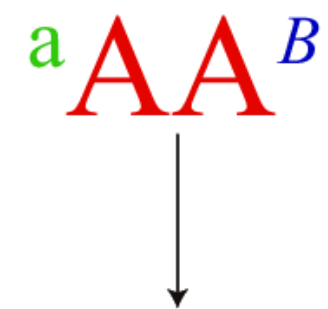

Huosang

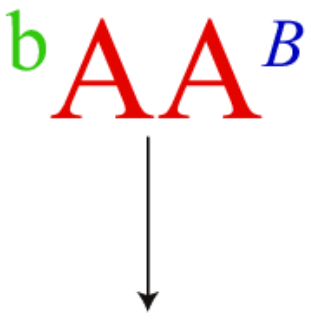

Lunjiao109

Clade 1, 2; clade II Pattern (3)
${ }^{b} \mathrm{BB}$

I

,

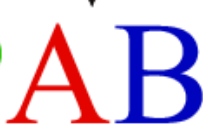

千

${ }^{b} \mathrm{BB}$

Yun6muben

Clade 3;

clade II, III

Pattern (2)

A $=$ short-type ITS $\quad \mathrm{a}=\mathrm{cpDNA}$ in clade I $\quad B=$ ITS pseudogene sequences

$\mathrm{B}=$ long-type ITS $\mathrm{b}=\mathrm{cpDNA}$ in clade II 


\section{Table $\mathbf{1}$ (on next page)}

Mulberry accessions used in this study and sequence characteristics of ITS, trnL-trnF, and trnT-trnL. 
1 Table 1. Mulberry accessions used in this study and sequence characteristics of ITS, $\operatorname{trn} L-\operatorname{trn} F$, and $\operatorname{trn} T$-trnL

\begin{tabular}{|c|c|c|c|c|c|c|c|c|c|c|c|}
\hline No. & Accessions & Taxa & $\begin{array}{l}\text { Ploidy } \\
\text { levels }\end{array}$ & $\begin{array}{c}\text { ITS length } \\
\text { (bp)\# }\end{array}$ & $\begin{array}{c}\text { ITS } \\
\text { heterozygosity }\end{array}$ & Accession No. (ITS) & $\begin{array}{l}\text { Clone } \\
\text { number }\end{array}$ & $\begin{array}{c}\text { trnL-trnF } \\
\text { (bp) }\end{array}$ & $\begin{array}{c}\text { Accession } \\
\text { No. }\end{array}$ & $\begin{array}{c}\text { trnT-trnL } \\
\text { (bp) }\end{array}$ & $\begin{array}{c}\text { Accession } \\
\text { No. }\end{array}$ \\
\hline 1 & Agentingsang & M. alba & $4 \mathrm{x}$ & $611 / 624$ & $40 \%$ & MN044824/MN044849 & 20 & 921 & MN057958 & 1,096 & MN057991 \\
\hline 2 & Banqiao6 & M. alba & $4 \mathrm{x}$ & 611 & $0 \%$ & MN044817 & 7 & 921 & MN057959 & 1,078 & MN057992 \\
\hline 3 & Basailuona & M. alba & $4 \mathrm{x}$ & 611 & $0 \%$ & MN044831 & 7 & 921 & MN057961 & 1,078 & MN057994 \\
\hline 4 & Gailiang10 & M. alba & $4 \mathrm{x}$ & 611 & $0 \%$ & MN044828 & 7 & 921 & MN057975 & 1,089 & MN057808 \\
\hline 5 & Hanguodabaizhenzhu & M. alba & $4 \mathrm{x}$ & $611 / 611$ & $14.29 \%$ & MN044825/MN044851 & 7 & 921 & MN057965 & 1,118 & MN057998 \\
\hline 6 & Huasang & M. alba & $4 \mathrm{x}$ & 611 & $0 \%$ & MN044813 & 7 & 921 & MN057967 & 1,078 & MN057800 \\
\hline 7 & Huai302 & M. alba & $12 \mathrm{x}$ & 611 & $0 \%$ & MN044836 & 7 & 921 & MN057966 & 1,078 & MN057999 \\
\hline 8 & Huosang & M. alba & $4 \mathrm{x}$ & $611 / 624$ & $5.26 \%$ & MN044833/MN044850 & 19 & 921 & MN057968 & 1,078 & MN057801 \\
\hline 9 & Jianpuzai & M. alba & $6 \mathrm{x}$ & $611 / 625$ & $10 \%$ & MN044834/MN044854 & 10 & 921 & MN057969 & 1,112 & MN057802 \\
\hline 10 & Leshandahongpi & M. alba & $6 x$ & 611 & $0 \%$ & MN044823 & 7 & 921 & MN057971 & 1,111 & MN057804 \\
\hline 11 & Lunjiao109 & M. alba & $4 \mathrm{x}$ & $611 / 624$ & $25.00 \%$ & MN044822/MN044847 & 16 & 921 & MN057972 & 1,118 & MN057805 \\
\hline 12 & Shanxitiansang & M. alba & $4 \mathrm{x}$ & $611 / 624$ & $5 \%$ & MN044832/MN044846 & 20 & 921 & MN057976 & 1,079 & MN057809 \\
\hline 13 & Shimiansang & M. alba & $4 \mathrm{x}$ & $611 / 611$ & $14.29 \%$ & MN044818/MN044845 & 7 & 922 & MN057977 & 1,079 & MN057810 \\
\hline 14 & Shuisang & M. alba & $4 \mathrm{x}$ & $611 / 611$ & $14.29 \%$ & MN044821/MN044835 & 7 & 921 & MN057978 & 1,117 & MN057811 \\
\hline 15 & Sililanka & M. alba & $4 \mathrm{x}$ & 611 & $0 \%$ & MN044830 & 7 & 921 & MN057980 & 1,078 & MN057813 \\
\hline 16 & Taiwanchaochangguo & M. alba & $4 \mathrm{x}$ & 611 & $0 \%$ & MN044820 & 7 & 921 & MN057981 & 1,119 & MN057814 \\
\hline 17 & Wupisang & M. alba & $4 \mathrm{x}$ & 611 & $0 \%$ & MN044814 & 7 & 922 & MN057982 & 1,078 & MN057815 \\
\hline 18 & Xinjiaposijiguosang & M. alba & $4 \mathrm{x}$ & 611 & $0 \%$ & MN044827 & 7 & 921 & MN057979 & 1,118 & MN057812 \\
\hline 19 & Xinyizhilai & M. alba & $4 \mathrm{x}$ & $611 / 624$ & $20 \%$ & MN044829/MN044848 & 20 & 921 & MN057983 & 1,078 & MN057816 \\
\hline 20 & Yidachimu & M. alba & $4 \mathrm{x}$ & 611 & $0 \%$ & MN044826 & 7 & 921 & MN057985 & 1,077 & MN057818 \\
\hline 21 & Zhenzhubai & M. alba & $4 \mathrm{x}$ & 611 & $0 \%$ & MN044819 & 7 & 922 & MN057988 & 1,118 & MN057821 \\
\hline 22 & Baojing7 & M. cathayana & $12 \mathrm{x}$ & $611 / 626$ & $5 \%$ & MN044838/MN044852 & 20 & 922 & MN057960 & 1,112 & MN057993 \\
\hline 23 & Gui23 & M. cathayana & $12 \mathrm{x}$ & 611 & $0 \%$ & MN044837 & 7 & 921 & MN057964 & 1,112 & MN057997 \\
\hline 24 & Pisang2 & M. cathayana & $18 \mathrm{x}$ & $611 / 611$ & $14.29 \%$ & MN044839/MN044844 & 7 & 921 & MN057974 & 1,112 & MN057807 \\
\hline 25 & Jimengsang & M. mongolica & $4 \mathrm{x}$ & 611 & $0 \%$ & MN044815 & 7 & 921 & MN057970 & 1,078 & MN057803 \\
\hline 26 & Mengsang & M. mongolica & $4 \mathrm{x}$ & 611 & $0 \%$ & MN044816 & 7 & 922 & MN057973 & 1,078 & MN057806 \\
\hline
\end{tabular}




\begin{tabular}{|c|c|c|c|c|c|c|c|c|c|c|c|}
\hline 27 & M. nigra & M. nigra & $44 \mathrm{x}$ & 624 & $0 \%$ & KF784875 & 7 & 922 & MN057984 & 1,105 & MN057817 \\
\hline 28 & M. notabilis & M. notabilis & $2 \mathrm{x}$ & 631 & $0 \%$ & KF784877 & 7 & 918 & MN057962 & 1,092 & MN057995 \\
\hline 29 & Yun6 & M. wittiorum & $5 \mathrm{x}$ & $611 / 625$ & $45 \%$ & MN044840/MN044856 & 20 & 921 & MN057986 & 1,112 & MN057819 \\
\hline 30 & Yun6muben & M. wittiorum & $4 \mathrm{x}$ & $625 / 624$ & $14.29 \%$ & MN044855/MN044843 & 7 & 921 & MN057989 & 1,112 & MN057822 \\
\hline 31 & Yun7 & M. wittiorum & $7 \mathrm{x}$ & $611 / 626$ & $5 \%$ & MN044842/MN044853 & 20 & 921 & MN057987 & 1,098 & MN057820 \\
\hline 32 & Yun7muben & M. wittiorum & $8 \mathrm{x}$ & 611 & $0 \%$ & MN044841 & 7 & 921 & MN057990 & 1,098 & MN057823 \\
\hline 33 & M. yunnanensis & $\begin{array}{c}M . \\
\text { yunnanensis }\end{array}$ & $2 \mathrm{x}$ & 631 & $0 \%$ & KF850474 & 7 & 918 & MN057963 & 1,094 & MN057996 \\
\hline
\end{tabular}

2 \#: ITS- $\alpha, \alpha 1$ and Y6MB- $\beta 1$ are showed ahead in the column of ITS length. 